\title{
LIII. Collision of $\alpha$ particles with light atoms III. Nitrogen and oxygen atoms
}

\section{Professor Sir E. Rutherford F.R.S.}

To cite this article: Professor Sir E. Rutherford F.R.S. (1919) LIII. Collision of $\alpha$ particles with light atoms III. Nitrogen and oxygen atoms, Philosophical Magazine Series 6, 37:222, 571-580, DOI: $10.1080 / 14786440608635918$

To link to this article: http://dx.doi.org/10.1080/14786440608635918

$$
\text { 曲 Published online: } 08 \text { Apr } 2009 .
$$

Submit your article to this journal $₫$

山 Article views: 27

Q View related articles $₫$

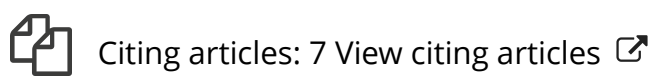


The calculated numbers are the sum of each observation worked out separately.

It will be seen that while there is a very satisfactory agreement between theory and experiment for the $a$ rays from polonium, the agreement is not so good for the $\mathrm{H}$ atoms. In the case of the $a$ rays, the number of doubles shows that the eye cannot distinguish an interval less than 1/10 second; while in the case of $\mathrm{H}$ atoms the number of doubles is nearly twice the theoretical number calculated on this power of distinction. Whether this difference is apparent or real is difficult to decide, for it must be remembered that counting such weak scintillations and at the same time distinguishing time intervals make a difficult task.

It is clear that under the experimental conditions, only a small fraction of the number of scintillations can be regarded as possible instantaneous doubles, and the effect is too small and uncertain to draw any very definite conclusions. It may be urged that a question of this kind could be settled more definitely by arranging that a small number of scintillations fell on the screen per minute when the probability of short intervals becomes very small. On the other hand, it takes a long time to count a sufficient number to compare theory with experiment, and it is very fatiguing to the eye and unreliable to count for long under such conditions.

I am much indebted to Professor Marsden for his valuable help in obtaining and analysing data for me on this important point.

LIII. Collision of a Particles with Light Atoms. III. Nitrogen and Oxygen Atoms. By Professor Sir E. Rutherford, F.R.S.*

$\mathbf{B}^{\mathrm{OHR} \dagger \text { has worked out a general theory of the absorp- }}$ B tion of electrified atoms in passing through matter, and has verified his conclusions by consideration of the absorption of a particles. On this theory, Darwin $\ddagger$ has shown that the range of a swift hydrogen atom in hydrogen can be calculated, and the value so found is in good accord with experiment. It is not difficult to deduce by the same method that the range $x$ in hydrogen of an electrified atom of charge $e$ and mass $m$ moving with a speed equal to an

* Communicated by the Author.

+ Bohr, Phil. Mag. xxv. p. 10 (1913).

$\ddagger$ Darwin, Phil. Mag. xxvii. p. 499 (1914).

2 R 2 
$\alpha$ particle of range $\mathrm{K}$ in hydrogen is given by

$$
x / \mathrm{R}=\frac{m}{\mathrm{M}} \cdot \frac{\mathrm{E}^{2}}{e^{2}},
$$

where $M$ is the mass and $E$ the charge on the $\alpha$ particle.

It is to be expected that this relation would hold approximately for the passage of electrified atums through light substances like air and aluminium. Since $\mathrm{M}=4$ and $\mathrm{E}=2 e$ where $e$ is the unit charge, the range $x$ of a particle carrying a single charge is obvionsly $x=m \mathrm{R}$. The velocity $u$ acquired by an atom of mass $m$ due to a close collision with an a particle of velocity $v$ is given by

$$
u=\frac{2 \mathrm{M}}{\mathrm{M}}+\vec{m} \cdot v \cos \theta,
$$

where $\theta$ is the angle of deflexion of the atom after the collision. Assuming that the range of electrified atoms in general like the range of a particles varies as the cube of the velocity, the range $x$ after collision of an atom carrying unit charge is given by

$$
x=m \mathrm{R}\left(\frac{2 \mathrm{M}}{\mathrm{M}+m}\right)^{3} \cos ^{3} \theta .
$$

Applying this result to $\mathrm{H}$ atoms, the maximum velocity should be $(8 / 5)^{3} \mathrm{R}=4 \cdot 1 \mathrm{R}$, while the observed value is about 4R. As a further test of this relation, consider the range to be expected for the recoil atom of radium B of mass $m$ resulting from the expulsion of an a particle of range $4.75 \mathrm{~cm}$. from radium $A$. By the principle of momentum $\mathrm{M} v=m u$ and the velocity of recoil $u=\frac{\mathrm{M}}{m} v$ where $m=214$. Consequently the range in air $x$

$$
=214 \cdot\left(\frac{4}{214}\right)^{3} \times 4 \cdot 75=\cdot 067 \mathrm{~cm} \text {. }
$$

The value found by Wertenstein * is $\cdot 12 \mathrm{~mm}$., but, considering the very wide range of velocity, the agreement is fairly satisfactory. If it be assumed that the range is proportional to the power $2 \cdot 85$ instead of 3 , this is a good agreement both for the hydrogen and recoil aloms.

If the atom after collision with an $\alpha$ particle carries a charge of two units, its range from (1) should be only about $1 / 4$ of the same atom carrying a single charge. For

* Wertenstein, C. R. cl. p. 869 (1910) ; cli. p. 469 (1910). 
example, in a collision of an o particle with the helium nucleus of equal mass, the range of the helium atom should be the same as the $\alpha$ particle before the collision if it carries two charges, but four times this range if it carries one charge.

We have collected in the following table data connected with the collision of $\alpha$ particles with the lighter atoms of matter. The moximum velocity, momentum, and energy of the atom after collision are given as fractions of that of the incident $\alpha$ particle. The range is calculated on the assumption that it is proportional to the power 2.9 of the velocity and that the atom carries unit charge.

For convenience, the data for hypothetical atoms of mass 2 and 3 times that of hydrogen are included.

\section{TABLE I.}

\begin{tabular}{|c|c|c|c|c|c|}
\hline Element. & $\begin{array}{l}\text { Atornic } \\
\text { weight. }\end{array}$ & $\begin{array}{l}\text { Ratio of } \\
\text { velocity } \\
\text { to that of } \\
\text { a particle. }\end{array}$ & $\begin{array}{l}\text { Ratio of } \\
\text { inomentum } \\
\text { to that of } \\
\text { a particle. }\end{array}$ & $\begin{array}{l}\text { Ratio of } \\
\text { energy } \\
\text { to that of } \\
a \text { particle. }\end{array}$ & $\begin{array}{l}\text { Ratio of } \\
\text { range } \\
\text { to that of } \\
\text { incident } \\
\alpha \text { particle. }\end{array}$ \\
\hline Hydrogen ..... & 1 & $1 \cdot 6$ & $\cdot 4$ & .64 & $3 \cdot 91$ \\
\hline$\quad ? \quad \ldots$ & 2 & $1 \cdot 33$ & $\cdot 66$ & $\cdot 89$ & $4 \cdot 6$ \\
\hline$? \quad \ldots \ldots \ldots$ & 3 & $1 \cdot 14$ & .85 & 98 & $5 \cdot 05$ \\
\hline Helium ........... & 4 & $I \cdot 00$ & 1.00 & 1.00 & 4.00 \\
\hline Jithium ............. & 7 & 727 & 1.27 & .925 & 278 \\
\hline Beryllium ......... & 9 & 615 & $1 \cdot 38$ & 85 & $\mathbf{2} \cdot 20$ \\
\hline Boron.............. & 11 & $\cdot 533$ & $1 \cdot 46$ & 78 & 1.77 \\
\hline Carbon ............. & 12 & .500 & 1.50 & $\cdot 75$ & $1 \cdot 61$ \\
\hline Nitrogen $\quad . . . . . .$. & 14 & $\cdot 444$ & 1.55 & 69 & $1 \cdot 33$ \\
\hline Oxygen ............ & 16 & $\cdot 400$ & 1.60 & $\cdot 64$ & $1 \cdot 12$ \\
\hline Fluorine........... & 19 & $\cdot 348$ & $1 \cdot 65$ & $\cdot 575$ & .89 \\
\hline Neon ................ & 20 & $\cdot 333$ & $1 \cdot 67$ & $\cdot 55$ & 82 \\
\hline Sodium ............ & 23 & 296 & 1.70 & 50 & .67 \\
\hline Magnesium ..... & 24 & .286 & $1 \cdot 71$ & $\cdot 49$ & $\cdot 64$ \\
\hline Aluminium ..... & 27 & $\cdot 258$ & 1.74 & .45 & .53 \\
\hline Iron ................ & 56 & $\cdot 133$ & 1.86 & .25 & $\cdot 19$ \\
\hline Silver.............. & 108 & 071 & 1.92 & $\cdot 136$ & .05 \\
\hline Gold ............... & 197 & .040 & $1 \cdot 92$ & $\cdot 079$ & $\cdot 017$ \\
\hline
\end{tabular}

It is seen that, on the assumption of unit charge, all the atoms of atomic weight up to oxygen should be detected beyond the range of the a particle. Supposing that $\alpha$ particles of range $7 \mathrm{~cm}$. are used, the maximum range to be expected for unit charge are for $\mathrm{He} 28 \cdot 0$, Li $19 \cdot 6$, Be $15 \cdot 4$, Bo $12 \cdot 4, C 11 \cdot 2, \mathrm{~N} \mathrm{9.3}, 07 \cdot 8 \mathrm{~cm}$.

Some preliminary experiments have been made with helium, using the apparatus similar to that employed for hydrogen and described in paper I. but on a smaller scale. 
The results show that if the collisions of $\alpha$ particles with helium atoms give any long-range scintillations of the order of $28 \mathrm{~cm}$. range, their number is very small compared with that produced in hydrogen under similar conditions. We may consequently conclude that the swift helium atoms produced by collision carry a double charge like the a particle.

A few experiments have been made to test whether the atoms of lithium, boron, or beryllium have the range to be expected if they carry a single charge. The salts $\mathrm{Lid}_{2} \mathrm{CO}_{3}$, $\mathrm{B}_{2} \mathrm{O}_{3}, \mathrm{~B} \theta \mathrm{O}$ were spread in a thin layer over the active source which was inclined at a small angle with the horizontal, and determinations made of the number of scintillations beyond the range of the $a$ particle. The air was exhausted and the $\alpha$ particles absorbed in aluminium and silver foils. No certain evidence was obtained of the presence of appreciable numbers of scintillations at the ranges to be expected if the atoms carry a single charge. Experiments of this kind are not easy on account of the difficulty of obtaining thin uniform films of the salts or metal under examination, and of the necessity of getting rid of all traces of hydrogen and water vapour, which give rise to numerous $H$ atoms. It is intended later to make a systematic examination of these elements to determine the range of the atoms produced by close collisions with $\alpha$ particles.

\section{Experiments in Air and Oxygen.}

Experiments on the range of swift atoms become much easier and more certain when the elements are in the gaseous state, for there is then no uncertainty with regard to the uniformity of the absorbing column and usually no difficulty in ensuring absence of hydrogen and water vapour. Thin films of rolled metals like aluminium, silver, or gold are usually very irregular in thickness. This irregularity comes out very obviously when intense sources of radiation are employed under conditions when one in a million of the incident particles can be detected. It is not unusual in these cases to find that $\alpha$ particles can be detected at a distance 10 per cent. beyond the average range of the a particles as determined by ordinary methods. Mica films are very uniform and show none of these irregularities, but unfortunately mica contains both hydrogen and oxygen and gives rise to numerous $\mathrm{H}$ and $\mathrm{O}$ atoms beyond the range of the bombarding a particles.

We have seeu that both $\mathrm{N}$ and $\mathrm{O}$ atoms carrying single charge should be detected beyond the range of the 
$\alpha$ particles, and this is borne out by experiment. In the case of air, the active disk conted with radium $C$ of $\gamma$-ray activity about $30 \mathrm{mg}$. Ra, was mounted with its plane vertical at a distance of about $7 \mathrm{~cm}$. from a zinc sulphide screen in the open air. Both the source and screen were placed between the poles of a large electromagnet to deflect the $\beta$ rays. The vertical convection currents due to the heated electromagnet prevented any contamination of the screen by active matter escaping from the source.

The end of the range of the a particles was sharply defined, but numerous bright scintillations were observed for distances nearly $2 \mathrm{~cm}$. beyond the range of the $\alpha$ particles. There was a steady decrease both in number and brightness up to $9 \mathrm{~cm}$. of air, and beyond that distance the small number of scintillations observed, due to $H$ atoms from the source and from the water vapour in the air, fell off slowly.

The range of these atoms was best determined by placing the screen just outside the range of the $\alpha$ particles $(7 \cdot 1 \mathrm{~cm}$. at $15^{\circ} \mathrm{C}$.) and then adding thin screens of aluminium foil close to the zinc sulphide screen. The variation of number with absorption in terms of cms. of air is shown in fig. 1 .

Fig. 1.

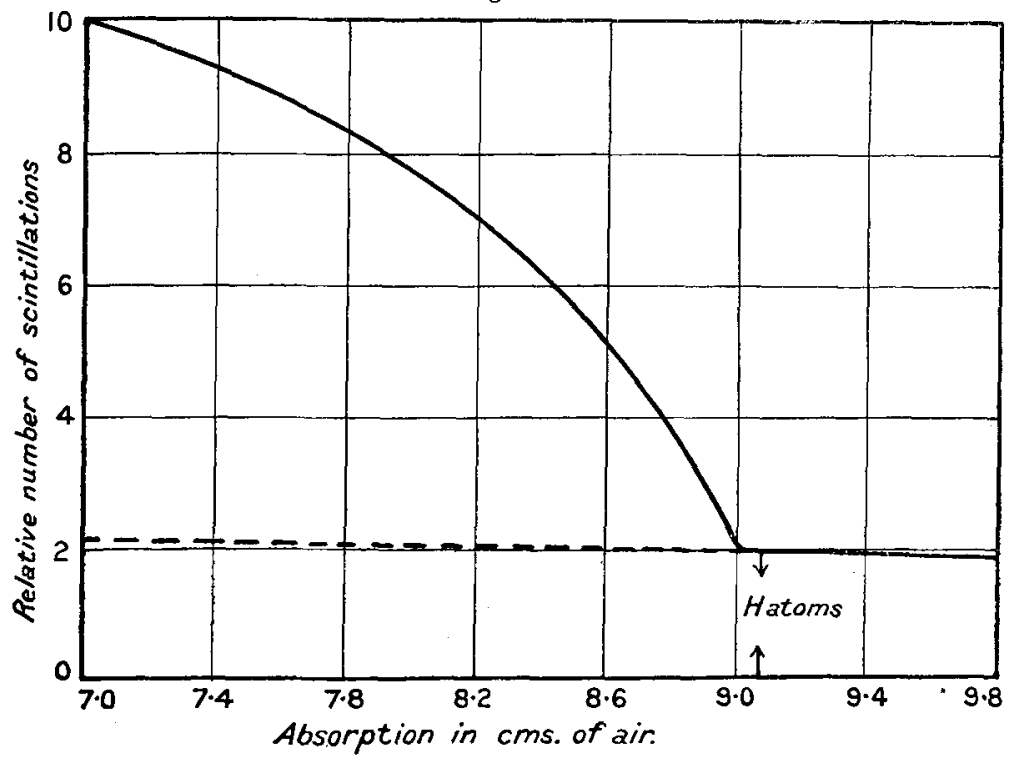

It will be seen that the scintillations fall off at first slowly with increase of absorption and more rapidly near the end of their range, which was equal to $9.0 \mathrm{~cm}$. of air at normal 
pressure and $15^{\circ} \mathrm{C}$. The scintillations, presumably due to swift $N$ and $O$ atoms, are bright and easily counted for a total absorption corresponding to about $7 \cdot 5 \mathrm{~cm}$. of air. At this stage they appear equal in brightness to those given by an a particle of range about $1 \mathrm{~cm}$.

In other experiments with air, nitrogen, and oxygen, and earbon dioxide, the screen and source were placed in a rectangular box and a slow current of the dried gas passed through during the experiment. 'This prevented contamination of the screen by diffusion of active matter from the source, and the range was determined by altering the distance between source and screen:

The scintillations in pure oxygen and carbon dioxide were about the same brightness for corresponding ranges, and had nearly the same equivalent ranges in air as those due presumably to $\mathrm{N}$ atoms from the air.

This was rather surprising, as we should expect the $O$ atoms to have considerably. less range than the lighter $\mathrm{N}$ atom. The calculated ranges (see table above) are $7 \cdot 8$ and $9 \cdot 3 \mathrm{~cm}$. respectively. This suggested the possibility that the scintillations might be due not to $N$ or $O$ atoms but to actual $\alpha$ particles of range $9 \mathrm{~cm}$. which were expelled from the radioactive source. If this were the case, the total rànge of the $\alpha$ partigles should not be altered by placing an absorbing screen of aluminium or gold of known stopping power close to the source in the path of the a rays. On the other hand, if the scintillations were due to swift $\mathrm{N}$ or $\mathrm{O}$ atoms from the air, the range should be diminished. For example, if a screen of stopping power equal to $3.5 \mathrm{~cm}$. of air were placed in the path of the $\alpha$ rays of range $7.0 \mathrm{~cm}$., the resulting range of the $\alpha$ particles acting on the gas is $3.5 \mathrm{~cm}$, and the total range of the $N$ or $O$ atoms measured from the sonrce should be $3 \cdot 5+\frac{8}{7} \times 3 \cdot 5=8 \cdot 0 \mathrm{~cm}$. instead of $9.0 \mathrm{~cm}$. Experiments of this kind were made with an aluminium and a gold screen of stopping powers $3 \cdot 7$ and $1 \cdot 2 \mathrm{~cm}$. respectively, but were not altogether satisfactory on account of the inequalities of the films already referred to. They showed, however, that no appreciable number of scintillations could be detected beyond $8 \mathrm{~cm}$. The results indicated that the scintillations were due to atoms of $\mathrm{N}$ and $O$ and not to $\alpha$ particles from the source. This was further confirmed by experiments with mica screens of stopping power $7 \cdot 0 \mathrm{~cm}$. The number of bright scintillations which resembled $\alpha$ particles were less than half the number observed in air or oxygen gas under similar conditions, but the presence of numerous $H$ atoms from the mica interfered 
with an accurate determination. Since mica contains oxygen as well as hydrogen we should obtain swift $O$ atoms, and the number of scintillations observed was about that to be expected from the amount of oxygen present, but was less than the number observed in air. There appears to be no doubt that the scintillations observed in air between the ranges 7 and $9 \mathrm{~cm}$. arise from collision of $\propto$ particles with $\mathrm{N}$ and $\mathrm{O}$ atoms. The observation that the range of the swift atoms, produced by $\alpha$ particles in their passage through carbon dioxide, is equivalent to the range of $O$ atoms, indicates that there are no carbon atoms carrying a single charge, for in that case bright scintillations should have been observed for ranges up to $12 \mathrm{~cm}$. of air (see Table I.).

It will be remembered that in the beautiful photographs of Mr. C.T.R. Wilson * showing the trails of $\alpha$ particles, an example is given where the $\alpha$ particle in air shows a sudden deflexion of $43^{\circ}$, and there is clear evidence of a well-marked spur presumably showing the trail of the $N$ or $O$ recoil atom. It is of interest to compare the length of this spur with the range to be expected for a collision with an $O$ atom. If $\phi$ be angle of deflexion of the $\alpha$ particle and $\theta$ the deflexion of the $\mathrm{O}$ atom,

$$
\tan \phi=\frac{m \sin 2 \theta}{M-m \cos 2 \theta}
$$

where $m=$ mass of $\mathrm{O}$ atom and $\mathrm{M}=$ mass of $\alpha$ particle.

Putting $\mathrm{M}=4, m=16, \phi=43^{\circ}$, then $\theta=63^{\circ} .55$.

If $v=$ velocity of the $\alpha$ particle before the collision, the velocity of the $O$ atom

$$
=\frac{2 \mathrm{M}}{\mathrm{M}+m} \cdot v \cos \theta=178 v
$$

while the velocity of the a particle after the collision is $\cdot 934 v$.

$$
\frac{\text { Range of recoil } O \text { atom }}{\text { Range of } \alpha \text { particle after collision }}=16 \times\left(\frac{\cdot 178}{-934}\right)^{2 \cdot 9}=\cdot 13 \text {. }
$$

This is based on the calculation that the maximum range of $O$ atoms due to $\alpha$ particles from radium $C$ is $7.8 \mathrm{~cm}$., while the observed range is $9.0 \mathrm{~cm}$. Making this correction, the value $\cdot 13$ becomes $\cdot 15$.

It is possible to compare only roughly the ranges of the $\alpha$ particle and recoil atom in the photograph, but the results are in fair accord with the calculation.

* C. T. R. Wilson, Proc. Roy. Soc. A. lxxx vii. p. 277 (1912). 
In the same photograph the $\alpha$ particle shows another sudden bend of $10^{\circ} 5$. In this case, the range of the recoil $O$ atom should be only about $1 / 800$ of the a particle and could not be distinguished on the photograph.

\section{Number of $\mathrm{N}$ atoms.}

In a previous paper we have calculated the number and distribution of $H$ atoms produced by $\alpha$ particles on the assumption that the nuclei may be regarded as point centres of force repelling according to the law of the inverse square. When these calculations are applied to the collision of a particles with nitrogen or oxygen nuclei, the distribution with velocity of the $\mathrm{N}$ and $\mathrm{O}$ atoms is very similar to that for $\mathrm{H}$ atoms. We should consequently expect on the simple theory that the number of $N$ and $O$ atoms should fall off very rapidly between 7 and $9 \mathrm{~cm}$., and that the number of short-range atoms should greatly preponderate. Quite the contrary is observed in the experiments (fig. 1), where it is seen that the number of scintillations fall off quite gradually with range.

There seems to be no doubt that the effects produced by the collision of a particles with $\mathrm{N}$ and $\mathrm{O}$ atoms are very similar to those observed in hydrogen. The observations only receive an explanation on the assumption that the $\mathrm{N}$ and $O$ atoms like the $H$ atoms are thrown forward mainly in the direction of the $\alpha$ particles and, at any rate for swift $\alpha$ particles, the velocities of the recoil atoms are nearly uniform for a given velocity of the $\alpha$ particles. It should be pointed out that the experiments with air and oxygen differ in one respect from those with hydrogen. In the case of air the $\alpha$ particles are completely absorbed in the column of gas, while in the case of hydrogen the stopping power was usually equivalent to less than $1 \mathrm{~cm}$. of air. Consequently in the air experiments, the scintillations observed are due to $\mathrm{N}$ and $\mathrm{O}$ atoms which are produced by $a$ particles of all ranges between 7 and $0 \mathrm{~cm}$., and thus have a wide range of velocities.

A number of experiments were made by the use of absorbing-screens of aluminium and gold in order to determine the number of $\mathrm{N}$ and $\mathrm{O}$ atoms produced by a particles of different range. The result as a whole showed that, for example, the number produced in the first $3.5 \mathrm{~cm}$. of the range of the a particle from radium $\mathrm{C}$ was greater than in the last $3.5 \mathrm{~cm}$., but accurate deductions were vitiated by the lack of uniformity in thickness of the metal films.

A number of concordant measurements were made to fix 
the total number of scintillations observed in air for a known activity of the source. The number of scintillations per minute due to $\mathrm{N}$ and $O$ atoms at a distance of $7.5 \mathrm{~cm}$. in air at $15^{\circ} \mathrm{C}$. was $2 \cdot 2$ on an area of the zinc sulphide screen equal to $3 \cdot 14 \mathrm{sq} . \mathrm{mm}$. Referring to curve 1 , it is seen that the number corresponding to an abserption of $7 \mathrm{~cm}$. should be 2.6 and the number for $8 \mathrm{~cm}$. absorption 1.5 .

All those atoms of range equal to or greater than $8 \mathrm{~cm}$. must be produced in the first $3.5 \mathrm{~cm}$. of the path of the $\alpha$ rays ; for the 0 atoms produced by $\alpha$ particles of range $3.5 \mathrm{~cm}$. cannot travel further than $\gamma \mathrm{cm}$. from the source, and probably only a small fraction reach this distance owing to scattering and straggling.

For the purpose of calculation, suppose that the production of swift atoms is uniform over the first $355 \mathrm{~cm}$. of the range and that $\rho$ is the ratio of the number of swift atoms produced per $\mathrm{cm}$. of path to the number of a particles passing through the gas.

The number $Q$ of recoil atoms falling per second on the screen of area $\mathrm{A}$ after passing through $l \mathrm{~cm}$. of gas is given by

$$
\mathrm{Q}=\rho \cdot \frac{\mathrm{A} l \mathrm{~N}}{4 \pi r^{2}}
$$

where $\mathrm{N}$ is the total number of $\alpha$ particles emitted by the source per second $\left(3 \cdot 7 \times 10^{7}\right.$ per second per mg. Ra of activity) and $r$ is the distance of the source from the screen.

Putting

$$
\mathrm{Q}=\frac{1.5}{60}, \quad \mathrm{~A}=.0314 \mathrm{sq} . \mathrm{cm} ., \quad l=3.5 \mathrm{~cm} ., \quad r=7 \cdot 5 \mathrm{~cm},
$$

then the average value of $\rho=4 \cdot 3 / 10^{6}$.

When we take into consideration the well-known way in which the $\alpha$ particles fall off near the end of the range in consequence of scattering, it is obvious that the true value of $\rho$ is considerably greater than the above and is probably about $7 / 10^{6}$.

In the experiments with hydrogen, it was shown that $\rho=1 / 10^{5}$ about-a value not very different from that observed in these experiments. We may consequently conclude that about the same number of swift atoms are produced per centimetre of path by the passage of $\alpha$ particles through air, oxygen, and hydrogen. As in the case of hydrogen, it can be shown that all $\alpha$ particles, shot within a perpendicular distance $p=2.4 \times 10^{-13} \mathrm{~cm}$. of the atomic nucleus, give rise to swift atoms of nitrogen and oxygen. 
It is clear from these results that the nuclei under consideration can no longer be regarded as point charges for distances of approach of the order of the diameter of the electron. As far as experiment has so far gone, it is difficult to fix with certainty the distance at which the forces between the nuclei become abnormal, but a rough estimate can be made. Regarding the nuclei as point charges, the closest distance of approach in a collision is $1.9 \times 10^{-12} \mathrm{~cm}$. in the case of a hydrogen atom and $3.8 \times 10^{-13} \mathrm{~cm}$. in the case of the oxygen atom. Taking into account the close similarity of the effects produced by a particles in hydrogen and oxygen and the greater repulsive forces between the nuclei in the latter case, it seems probable that the abnurmal forces in the case of oxygen manifest themselves at about twice the distance observed in the case of hydrogen. This would mean that the rapid variation in the magnitude and direction of the forces between the nuclei which lead to the recoil of swift atoms mainly in the direction of the a particle should begin at a distance about $7 \times 10^{-13} \mathrm{~cm}$. Such a result is to be anticipated on general grounds, for presumably the oxygen nucleus is more complex and has larger dimensions than that of helium.

In a paper published three years ago Mr. A. B. Wood and the writer ${ }^{*}$ described experiments which showed that. the active deposit of thorium gave rise to a few a particles of range $11.3 \mathrm{~cm}$. in addition to the main group of ranges $5 \cdot 0$ and $8.6 \mathrm{~cm}$. In these experiments, the $\alpha$ rays of range $8.6 \mathrm{~cm}$. were absorbed in mica. In the light of the present experiments, the oxygen present in the mica should give rise to scintillations like $\alpha$ particles of range

$$
8 \cdot 6 \times \frac{9 \cdot 0}{7 \cdot 0}=1 \dot{1} \cdot 1 \mathrm{~cm} .
$$

This range is nearly the same as that observed in the thorium experiment, and raises the question whether these long range $\alpha$ particles are not in reality due to collision of a particles with the oxygen atoms in the mica. A fraction of the scintillations must undoubtedly have been due to this cause, but on the other hand the number of scintillations observed, about $1 / 10000$ of the number of a particles, is considerably greater than is to be expected from the experiments with radium C. Further experiments to clear up this important point have been undertaken by Professor Marsden in New Zealand. 